\title{
Use of the TELE-ASD-PEDS for Autism Evaluations in Response to COVID-19: Preliminary Outcomes and Clinician Acceptability
}

\author{
Liliana Wagner ${ }^{1,2}$ - Laura L. Corona ${ }^{1,2} \cdot$ Amy S. Weitlauf ${ }^{1,2} \cdot$ Kathryn L. Marsh $^{5} \cdot$ Anna F. Berman $^{1}$. \\ Neill A. Broderick ${ }^{1,2} \cdot$ Sara Francis $^{1,2}$. Jeffrey Hine ${ }^{1,2} \cdot$ Amy Nicholson $^{1,2,3} \cdot$ Caitlin Stone $^{1,2} \cdot$ Zachary Warren $^{1,2,3,4}$
}

Accepted: 21 October 2020 / Published online: 30 October 2020

(c) Springer Science+Business Media, LLC, part of Springer Nature 2020

\begin{abstract}
The COVID-19 pandemic has caused unprecedented disruptions to healthcare, including direct impacts on service delivery related to autism spectrum disorder (ASD). Caregiver-mediated tele-assessment offers an opportunity to continue services while adhering to social distancing guidelines. The present study describes a model of tele-assessment for ASD in young children, implemented in direct response to disruptions in care caused by the COVID-19 pandemic. We present preliminary data on the outcomes and provider perceptions of tele-assessments, together with several lessons learned during the period of initial implementation.
\end{abstract}

Keywords Autism spectrum disorder · Assessment · Young children · Telemedicine

The COVID-19 pandemic has caused unprecedented disruptions to healthcare and behavioral healthcare systems across the globe (Wong et al. 2020), resulting in delays in patient care and requiring many providers to consider alternative, novel means of delivering services. Within our university affiliated medical setting, in-person diagnostic evaluations for autism spectrum disorder (ASD) were among the services suspended as part of our institution's initial response to the pandemic. Given the prospect of growing wait lists and increasing delays in connecting families with needed services and resources, we implemented a telemedicinebased model of ASD assessment with an initial focus on children under 36 months of age. In what follows, we

Liliana Wagner

Liliana.Wagner@vumc.org

1 Vanderbilt Kennedy Center/Treatment and Research Institute for Autism Spectrum Disorders, Vanderbilt University Medical Center, 1207 17th Avenue, Suite 202, Nashville, TN 37203, USA

2 Department of Pediatrics, Vanderbilt University Medical Center, Nashville, TN, USA

3 Department of Psychiatry and Behavioral Sciences, Vanderbilt University Medical Center, Nashville, TN, USA

4 Department of Special Education, Vanderbilt University, Nashville, TN, USA

5 Unumb Center of Neurodevelopment, Columbia, SC, USA describe this model of care and present preliminary data on the outcomes and provider perceptions of tele-assessments, together with several lessons learned during the period of initial implementation.

Even before the disruptions due to COVID-19, there has been a growing need for novel models of care to address delays in accessing autism-specific diagnostic and intervention services (Zwaigenbaum and Warren 2020). Although a stable diagnosis of ASD is possible in the second year of life for many children (Corsello et al. 2013; Pierce et al. 2019), diagnostic delays persist, with the average age of diagnosis hovering around four years of age (Maenner et al. 2020). Myriad geographic, socioeconomic, and provider-level factors contribute to these diagnostic delays (Bishop-Fitzpatrick and Kind 2017; Mazurek et al. 2014). Experts in ASD diagnosis are scarce and have long wait lists (Hyman and Johnson 2012). Many providers are concentrated in metropoli$\tan$ areas, requiring some families to travel long distances. Gold-standard diagnostic assessments may take a full day or longer, necessitating that families miss work. These challenges disproportionately impact under-resourced families with financial constraints or geographic or language-related barriers (Antezana et al. 2017; Durkin et al. 2010; Khowaja et al. 2015).

One promising avenue for increasing access to early ASD assessment is telemedicine-based evaluation (Corona et al. 2020b; Juárez et al. 2018). Most existing work regarding 
tele-assessment of ASD relies upon having a trained provider present with the family in one location, while a remote psychologist converses with the family and observes the child's behavior (Juárez et al. 2018; Stainbrook et al. 2019). Additional preliminary work has explored tools for caregivermediated tele-assessment, in which caregivers complete assessment activities with their child, while being coached by a remote provider (Corona et al. 2020b). The COVID-19 pandemic has accelerated interest in tele-assessment, particularly methods that allow providers to complete remote assessments with families in their home settings (Narzisi 2020; Wright et al. 2020).

When considering telemedicine evaluations within the home, providers must address a multitude of associated logistical and ethical considerations (Joint Task Force for the Development of Telepsychology Guidelines for Psychologists 2013; Kanne and Bishop 2020). One must consider provider training needs and capacity as well as the ability of families to provide information or implement tasks. Tools used during telemedicine evaluations must be capable of home deployment using freely available, low cost toys and materials. Additionally, although a provider may deliver instructions remotely, the caregiver must be able and willing to administer the activities and provide real-time feedback on their children's responses while also managing the required technology and materials.

The TELE-ASD-PEDS (Corona et al. 2020a) is a tool specifically developed for caregiver-mediated telemedicine evaluation of ASD (clinicaltrials.gov, NCT03847337). This tool, designed for providers with expertise in the early diagnosis of ASD, facilitates the remote observation of ASD symptoms in young children based upon caregiver-mediated interactions, using readily available toys and materials found in most families' homes. When in-person clinical services were halted due to the pandemic, this tool was deployed by expert psychologists at our university-affiliated medical center for use within telehealth-to-home evaluations.

The goal of the current paper is to present our clinical response to the disruptions in care caused by the COVID19 pandemic, including a description of our model of teleassessment and data on the number of children seen through telehealth, diagnoses given, and factors contributing to diagnostic certainty. We also specifically examine the use of the TELE-ASD-PEDS as a tool for tele-assessment, as obtaining preliminary information on the acceptability and feasibility of the tool will inform future studies rigorously evaluating its psychometric properties. Current evaluation of the psychometric properties is underway (clinicaltrials. gov, NCT03847337), but due to extraordinary circumstance of the COVID-19 pandemic the use of the tool was initiated prior to our ability to adequately complete some properties of tool use (i.e. accuracy, reliability, concurrent validity data). Finally, we address lessons learned to be shared with other providers, including adjustments in our communication with families to prepare them for their appointments and shifts in the modalities used to provide recommendations and resources.

\section{Methods}

\section{Overview of Clinical Process}

Following suspension of in-person appointments, families scheduled or on the waitlist for a traditional comprehensive evaluation for ASD were contacted by a clinic scheduler and presented with the option of scheduling a telemedicine appointment. When contacting families, schedulers followed scripts that provided an overview of the tele-assessment process including technology requirements, estimated appointment time, recommended materials, a description of the caregiver's role, and possible outcomes of the assessment. No families were denied the opportunity for future in-person services if they declined telehealth evaluation; however, no in-person evaluations were available during this period nor were families provided with specific information about how/when future in-person evaluations would take place. After scheduling, families were provided with further written information about accessing the telehealth platform and preparing for the appointment. This information was delivered through the electronic medical record (EMR) system or via secure email, if families preferred. During telemedicine appointments, caregivers used a personal device with audiovisual capabilities (smart phone, tablet, laptop) to access a HIPAA-compliant Zoom video platform available through the medical center's EMR system. Clinicians used laptops with audio-visual capabilities to access the same system.

At the beginning of the appointment, remote clinicians greeted the families and provided an overview of the visit procedures, including discussion of the use of telemedicine, limits to confidentiality, limits of the clinical information to be gained relative to traditional in-person assessment (i.e., not obtaining estimates of other areas of developmental functioning, such as language or fine motor skills), and possible outcomes of tele-assessment (i.e., confirming ASD, ruling out ASD, recommending additional testing at a later date). Families provided verbal consent for telemedicine appointments. Verbal consent was documented by providers within the EMR in addition to formal consenting procedures within the EMR itself.

Each appointment included administration of the TELEASD-PEDS and a clinical interview with caregivers, including questions about the child's developmental history, current behaviors and skills in the home setting, and presence or absence of ASD-related behaviors. Clinicians combined the TELE-ASD-PEDS behavioral observations with other 
visit information (clinical interview, adaptive information, spontaneous child behaviors observed throughout the visit) to form a diagnostic impression (ASD vs. no ASD). At the conclusion of the appointment, clinicians shared their diagnostic impressions with the family and provided recommendations and resources. The majority of providers (67\%) reported spending between 60 and 120 min with a family during an average telemedicine visit; the remaining one third of providers reported spending 120-180 min with families. Following the appointment, clinicians sent evaluation reports and additional resources (e.g., handouts on ASD and interventions) to families either by mail, through the EMR, or encrypted HIPAA compliant email.

\section{Patient and Provider Characteristics}

Data analyzed for the present study include clinical data from 204 telemedicine evaluations using the TELE-ASDPEDS during approximately the first three months that teleassessments were implemented. As the tool was designed for children under three years of age, we restricted our analysis to evaluations with children between 16 and 36 months of age (mean age $=27.54$ months, $\mathrm{SD}=5.36$ months; see Table 1). The families included in this analysis were from the three states (TN, AL, KY) in which providers were licensed (or had received temporary emergency licensure) to provide telemedicine services.

All children included in these analyses had been referred for evaluation of developmental concerns related to ASD. Referral sources include Part C early intervention providers and community pediatricians. To be eligible for teleassessment, the family needed to live in a state in which providers were licensed to provide services, have access to a stable internet connection, and ensure a primary caregiver was available for the entire visit to provide information. Children with a severe sensorimotor impairment (i.e., hearing or vision impairment not able to be corrected, child not yet walking) that would impact participation in assessment activities were not eligible for tele-assessment.

Remote clinicians $(n=9)$ were licensed clinical psychology providers with expertise diagnosing ASD in young children. Clinicians had an average of eight years of experience
( $\mathrm{SD}=6.14$ years; range $=2-20$ years $)$ working in a pediatric setting with children with ASD. All clinicians were research reliable on the Autism Diagnostic Observation Schedule, Second Edition (ADOS-2; Lord et al. 2012) and actively engaged in clinical practice related to ASD diagnosis prior to cessation of in-person clinical activities. Two of the clinicians reported using telemedicine in their clinical practice prior to the suspension of in-person appointments.

\section{Assessment Tools}

TELE-ASD-PEDS. The TELE-ASD-PEDS (Corona et al. 2020a) is a novel tool developed for the remote observation of autism-related behaviors in young children (clinicaltrials. gov, NCT03847337). It is designed to reduce the resource burden associated with diagnostic assessments by employing minimal training requirements, widely available materials, and a brief administration time (15-20 min). The TELEASD-PEDS was designed to elicit symptoms of ASD in children under 36 months of age in order to augment diagnostic decision-making (yes/no/maybe ASD) by an expert clinician who also administers a comprehensive developmental history and symptom-focused interview.

The TELE-ASD-PEDS is based upon the ASD-PEDS, an assessment procedure developed through the application of machine learning techniques to a large clinical database of several hundred children who had received gold standard evaluations for ASD (Adiani et al. 2019). Feature engineering was then used to identify the most predictive items $(n=7)$ across assessment tools, and a team of ADOS-2 research-reliable expert clinicians operationalized behavioral descriptors based on the underlying constructs of the items. The clinicians then generated a list of administration tasks intended for clinician use to facilitate observation of these behaviors.

As part of ongoing work (clinicaltrials.gov, NCT03847337), the ASD-PEDS was adapted by this same group of clinical providers such that clinicians could coach caregivers in a remote location to administer ASD-PEDS activities. This tool, the TELE-ASD-PEDS, includes eight discrete, caregiver-led activities or social bids, including opportunities for interactive play, physical play routines, and
Table 1 Participant demographics by diagnostic status

\begin{tabular}{|c|c|c|c|c|c|}
\hline & Full sample n (\%) & ASD diagnosed & ASD suspected & Diagnosis uncertain & No ASD \\
\hline \multicolumn{6}{|c|}{ Toddlers (n [\%]) } \\
\hline $\mathrm{N}$ & 204 & 145 & 14 & 22 & 23 \\
\hline $\begin{array}{l}\text { Age in } \\
\text { months } \\
(m[S D])\end{array}$ & $27.54(5.36)$ & $27.45(5.21)$ & $27.86(5.56)$ & $27.95(5.80)$ & $27.52(6.07)$ \\
\hline Male & $157(77 \%)$ & $112(77 \%)$ & $13(93 \%)$ & $17(77 \%)$ & $15(65 \%)$ \\
\hline Female & $47(23 \%)$ & $33(23 \%)$ & $1(7 \%)$ & $5(23 \%$ & $8(35 \%)$ \\
\hline
\end{tabular}


requesting activities. Modifications to the instructions and tasks from the original ASD-PEDS were minor and reflect the need to use materials that families have available in the home. For example, whereas the ASD-PEDS required the use of bubbles and a balloon, the TELE-ASD-PEDS offers suggestions for alternative objects/toys. In both the ASDPEDS and TELE-ASD-PEDS, the instructions and tasks can be modified and repeated as needed in order for the provider to make meaningful observations, and caregivers are often asked to confirm or clarify observations. Following administration, clinicians provide ratings on seven behaviors (e.g., socially directed speech and sounds, frequent and flexible eye contact, unusual or repetitive play), using both dichotomous (yes/no) and Likert $(3=$ behaviors characteristic of ASD clearly present; $2=$ behaviors characteristic of ASD present at subclinical levels; $1=$ behaviors characteristic of ASD not present) scoring procedures (see Table 2). Clinicians calculate a total score by summing the child's Likertstyle scores for each key behavior.

\section{Provider survey}

A provider survey designed explicitly for the present study assessed providers' use, perceptions of, and satisfaction with telemedicine procedures for ASD assessment.

\section{Data Collection}

Following each tele-assessment visit, clinicians completed the TELE-ASD-PEDS rating form to document (1) clinician diagnostic impression (ASD vs. no ASD vs. unsure), (2) the diagnosis assigned following the appointment, (3) a Likert rating of clinician diagnostic certainty $(1=$ completely uncertain, $2=$ somewhat uncertain, $3=$ somewhat certain, $4=$ completely certain), and (4) whether further in-person testing was recommended.

After approximately three months of direct-to-home teleassessment, participating providers were emailed a link to complete the provider survey and asked to share feedback on their use, perceptions of, and satisfaction with tele-assessment and the TELE-ASD-PEDS. The survey included places for providers to give qualitative feedback. This was analyzed by (a) identifying key themes and (b) quantifying the occurrence of those themes across providers.

\section{Results}

\section{Evaluation Outcomes}

Of the 204 children who participated in telediagnostic evaluations, $71 \%$ received diagnoses of ASD following evaluation (ASD Diagnosed). For seven percent of children, clinicians indicated that children likely met criteria for ASD based on diagnostic impression (i.e., selected 'ASD' in forced choice diagnostic impression), but diagnosis was deferred pending further evaluation (ASD Suspected). Clinicians reported being unsure of whether children met criteria for ASD in 11 percent of evaluations, in all cases deferring a diagnosis and referring for in-person testing (Diagnosis Uncertain). Finally, $11 \%$ of children did not meet criteria for ASD (No ASD). These children were assigned diagnoses of developmental delay or speech/language delay $(n=5)$, anxiety $(n=1)$, or other specified disorder of psychological development $(n=17)$. Of these children, $48 \%$ were referred for further in-person evaluation. Mean TELE-ASD-PEDS scores and clinicians' mean ratings of diagnostic certainty are presented in Table 3.

One-way analysis of variance was conducted to compare TELE-ASD-PEDS total scores across groups. Due to violation of the assumption of homogeneity of variances (Levene's statistic $=4.72, p<0.05$ ), Welch's ANOVA was used (Delacre et al. 2019). The omnibus test of the model indicated that there were significant differences among TELEASD-PEDS scores (Welch's statistic $(3,38.1)=165.17$, $p<0.05)$. Post-hoc comparisons using the Games-Howell procedure indicated significant differences in TELE-ASDPEDS scores among all four groups (all p-values $<0.01$; See Table 4). Children who received an ASD diagnosis following tele-assessment received the highest TELE-ASD-PEDS scores $(\mathrm{m}=17.96, \mathrm{SD}=2.36)$, followed by children with suspected ASD $(m=15.14, \mathrm{SD}=2.45)$, then children with unclear diagnostic presentations $(\mathrm{m}=12.32, \mathrm{SD}=1.52)$. Children for whom ASD was ruled out received the lowest TELE-ASD-PEDS scores $(m=9.96, S D=1.64)$.

A second ANOVA was conducted to examine providers' diagnostic certainty across diagnostic category. Again, Welch's ANOVA was used due to violation of the homogeneity of variance assumption (Levene's statistic $=2.91$, $p<0.05)$. The overall test of the model demonstrated significant differences in diagnostic certainty (Welch's statistic $(3,32.70)=69.83, p<0.01)$. Post-hoc comparisons using the Games-Howell procedure indicated that providers reported higher levels of diagnostic certainty for children who received ASD diagnoses $(m=3.77, \mathrm{SD}=0.46)$ than for all other groups (all p-values $<0.01$ ). Providers reported higher diagnostic certainty for children in the No ASD group $(m=2.83, \mathrm{SD}=0.72)$ than children in the Diagnosis Uncertain group $(m=2.10, S D=0.61)$. For children with suspected ASD referred for in-person testing, provider diagnostic certainty did not differ from provider certainty in "No ASD" and "Diagnosis Uncertain" cases (See Table 5).

Finally, multiple linear regression was used to examine relations among child sex, age, TELE-ASDPEDS scores, and provider diagnostic certainty. Visual inspection of scatterplots indicated that child age and 


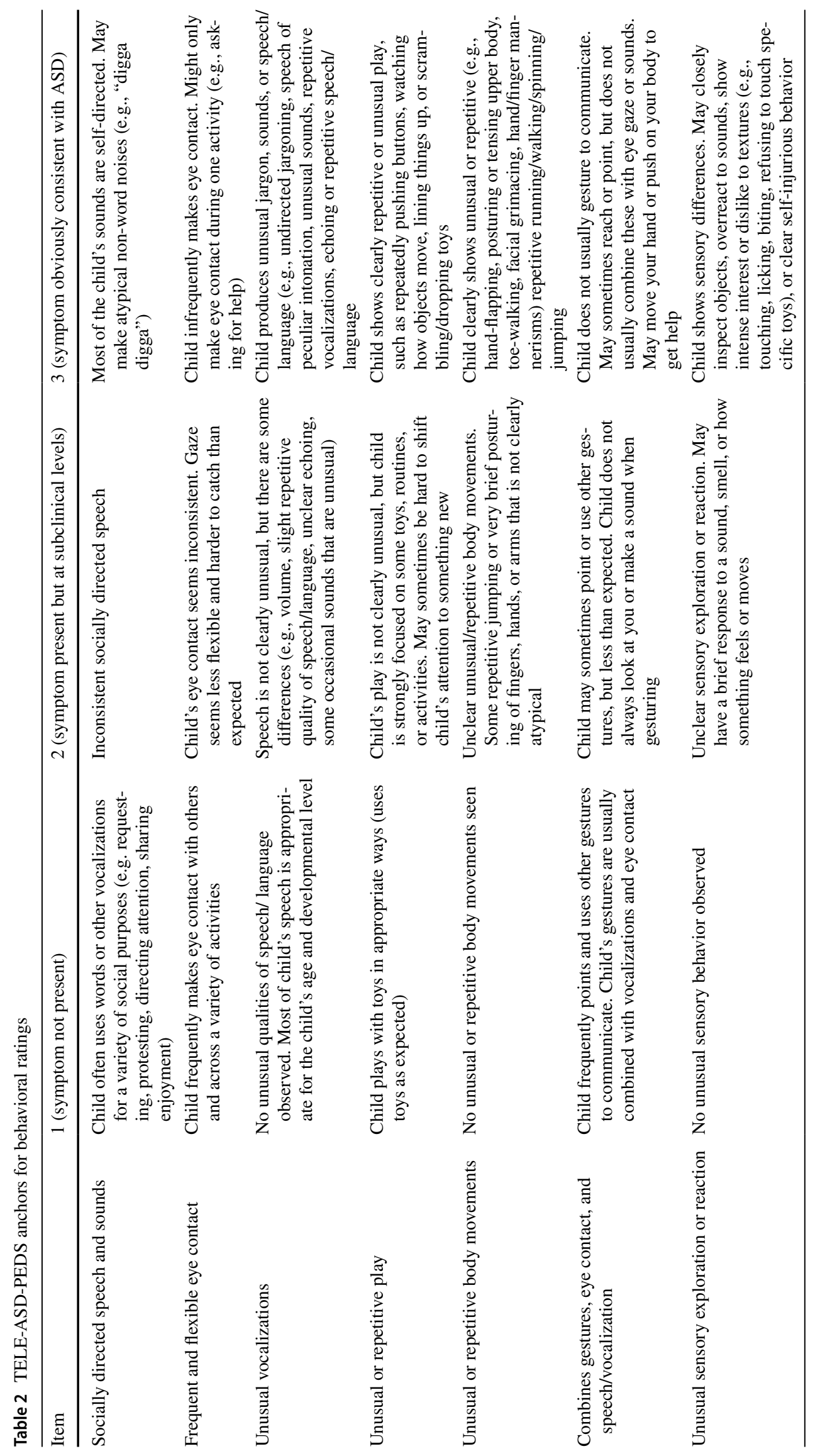


Table 3 Outcomes of telemedicine evaluation

\begin{tabular}{lllll}
\hline Clinician diagnostic impression & $\mathrm{N}(\%)$ & $\begin{array}{l}\text { TELE-ASD- } \\
\text { PEDS score* }\end{array}$ & $\begin{array}{l}\text { Further testing } \\
\text { recommended }\end{array}$ & $\begin{array}{l}\text { Clinician } \\
\text { diagnostic } \\
\text { certainty** } \\
m(S D)\end{array}$ \\
\hline ASD diagnosed & $145(71 \%)$ & $17.96(2.36)$ & $6 \%$ & $3.77(0.46)$ \\
ASD suspected & $14(7 \%)$ & $15.14(2.45)$ & $100 \%$ & $2.50(0.65)$ \\
Diagnosis uncertain & $22(11 \%)$ & $12.32(1.52)$ & $100 \%$ & $2.09(0.61)$ \\
No ASD & $23(11 \%)$ & $9.96(1.64)$ & $48 \%$ & $2.83(0.72)$ \\
\hline
\end{tabular}

*TELE-ASD-PEDS scores ranged from 7 to 21

${ }^{* *}$ Clinicians rated diagnostic certainty on a Likert scale, with $4=$ completely certain, $3=$ somewhat certain, $2=$ somewhat uncertain, and $1=$ completely uncertain

\begin{tabular}{llcclcc}
\hline Group & $\mathrm{N}$ & Mean & $\mathrm{SD}$ & \multicolumn{3}{l}{ Games Howell mean difference } \\
\cline { 5 - 7 } & & & & ASD suspected & $\begin{array}{l}\text { Diagnosis } \\
\text { uncertain }\end{array}$ & No ASD \\
\hline ASD diagnosed & 145 & 17.96 & 2.36 & $2.86^{*}$ & $5.64^{* *}$ & $8.00^{* *}$ \\
ASD suspected & 14 & 15.14 & 2.45 & - & $2.83^{*}$ & $5.19^{* *}$ \\
Diagnosis uncertain & 22 & 12.32 & 1.52 & & - & $2.36^{* *}$ \\
No ASD & 23 & 9.96 & 1.64 & & & - \\
\hline
\end{tabular}

p $<.01 ; * * \mathrm{p}<.001$

$1 *$ TELE-ASD-PEDS scores ranged from 7 to 21

\begin{tabular}{|c|c|c|c|c|c|c|}
\hline \multirow[t]{2}{*}{ Group } & \multirow[t]{2}{*}{$\mathrm{N}$} & \multirow[t]{2}{*}{ Mean $^{1}$} & \multirow[t]{2}{*}{ SD } & \multicolumn{3}{|c|}{ Games Howell mean difference } \\
\hline & & & & ASD suspected & $\begin{array}{l}\text { Diagnosis } \\
\text { uncertain }\end{array}$ & No ASD \\
\hline ASD diagnosed & 145 & 3.77 & 0.46 & $1.27 * *$ & $1.67 * *$ & $0.94 * *$ \\
\hline ASD suspected & 14 & 2.50 & 0.65 & - & 0.41 & -0.33 \\
\hline Diagnosis uncertain & 22 & 2.09 & 0.61 & & - & $-0.74^{*}$ \\
\hline No ASD & 23 & 2.83 & 0.72 & & & - \\
\hline
\end{tabular}

* $\mathrm{p}<.01 ; * * \mathrm{p}<.001$

${ }^{1}$ Clinicians rated diagnostic certainty on a Likert scale, with $4=$ completely certain, $3=$ somewhat certain, $2=$ somewhat uncertain, and $1=$ completely uncertain
TELE-ASD-PEDS scores linearly related to provider diagnostic certainty. Assumptions regarding homoscedasticity and normality of residuals were met. Tests for multicollinearity indicated no concern for multicollinearity (variance inflation factor [VIF] $<2$ for all predictors). The three-predictor model accounted for $43 \%$ of the variance in provider diagnostic certainty $(F(3,200)=50.74$, $p<0.01)$. TELE-ASD-PEDS scores emerged as the only significant predictor of diagnostic certainty $(\beta=0.65$, $\mathrm{t}=12.28, \mathrm{p}<0.01)$. Neither child $\operatorname{sex}(\beta=-0.22, \mathrm{t}=-0.42$, $\mathrm{p}=0.68)$ nor child age $(\beta=0.05, \mathrm{t}=0.88, \mathrm{p}=0.38)$ were significant predictors.

\section{Provider Perceptions}

Providers reported feeling comfortable completing teleassessments, making diagnoses of ASD following teleassessment, and providing feedback and recommendations to families via telemedicine (See Table 6). Providers also reported being satisfied with the TELE-ASD-PEDS and feeling comfortable guiding parents to complete the TELEASD-PEDS. All providers reported feeling that it was appropriate $(67 \%)$ or very appropriate $(33 \%)$ for toddlers to receive a diagnosis of ASD following tele-assessment. 
Table 6 Provider comfort and satisfaction with tele-assessment

\begin{tabular}{|c|c|c|c|c|c|c|}
\hline & Very comfortable & Comfortable & Mildly comfortable & $\begin{array}{l}\text { Mildly uncomfort- } \\
\text { able }\end{array}$ & uncomfortable & $\begin{array}{l}\text { Very } \\
\text { uncom- } \\
\text { fortable }\end{array}$ \\
\hline $\begin{array}{l}\text { How comfortable do you feel com- } \\
\text { pleting a telemedicine assessment } \\
\text { for a toddler with concerns for } \\
\text { autism? }\end{array}$ & $4(44 \%)$ & $4(44 \%)$ & $1(11 \%)$ & - & - & - \\
\hline $\begin{array}{l}\text { How comfortable do you feel making } \\
\text { a diagnosis of autism for a toddler } \\
\text { following a telemedicine assess- } \\
\text { ment? }\end{array}$ & $3(33 \%)$ & $5(56 \%)$ & $1(11 \%)$ & - & - & - \\
\hline $\begin{array}{l}\text { How comfortable do you feel discuss- } \\
\text { ing ASD diagnoses and providing } \\
\text { recommendations with families } \\
\text { during a telemedicine visit? }\end{array}$ & $4(44 \%)$ & $4(44 \%)$ & $1(11 \%)$ & - & - & - \\
\hline \multirow{2}{*}{$\begin{array}{l}\text { How comfortable were you walking a } \\
\text { caregiver through the TELE-ASD- } \\
\text { PEDS during a telemedicine visit? }\end{array}$} & $5(56 \%)$ & $4(44 \%)$ & - & - & - & - \\
\hline & Very helpful & Helpful & Neutral & Somewhat helpful & \multicolumn{2}{|c|}{ Not at all helpful } \\
\hline \multirow{2}{*}{$\begin{array}{l}\text { How helpful was the TELE-ASD- } \\
\text { PEDS in providing clinical informa- } \\
\text { tion to guide decision-making? }\end{array}$} & $6(67 \%)$ & $3(33 \%)$ & - & - & - & \\
\hline & Very satisfied & Satisfied & Neutral & Somewhat satisfied $\mathrm{N}$ & \multicolumn{2}{|l|}{ Not satisfied } \\
\hline $\begin{array}{l}\text { Please rate your satisfaction with the } \\
\text { TELE-ASD-PEDS }\end{array}$ & $5(56 \%)$ & $4(44 \%)$ & - & - & \multicolumn{2}{|c|}{-} \\
\hline
\end{tabular}

Providers were also asked for their qualitative feedback on elements of tele-assessment, including feedback on the TELE-ASD-PEDS, challenges encountered during tele-assessment, and perceived benefits of tele-assessment. Regarding the TELE-ASD-PEDS, four providers shared specific feedback on the administration guidelines and rating form. These included suggestions to simplify language and provide more concrete examples within the rating form; as well as challenges related to explaining particular activities (e.g., a "Ready-Set-Go" paradigm) to parents.

Regarding challenges experienced during tele-assessments, all providers reported experiencing technologyrelated challenges, including dropped calls, inconsistent audio, and challenges with helping caregivers to set up and adjust the camera throughout the appointment. Some providers $(n=5)$ also reported challenges related to the home environment (e.g., distractions, presence of multiple people with the caregiver and child, availability of play materials). One provider noted challenges related to obtaining and documenting informed consent for tele-assessment procedures. Providers also discussed challenges specifically related to the TELE-ASD-PEDS, including reliance on caregivers to share observations of eye contact and language use $(n=1)$, as well as individual differences among caregivers in their understanding of verbal instructions and ways in which they played or interacted with their children $(n=4)$.
Finally, when asked about benefits of tele-assessment, all providers described the value of observing children and families in their home environments. Providers also acknowledge the benefit of continuing to provide services during COVID-19-related restrictions $(n=4)$, as well as benefits to families in terms of limiting travel and transportation barriers $(n=6)$.

\section{Discussion}

The shift in clinical service delivery models necessitated by the COVID-19 pandemic provided an unexpected opportunity to evaluate a novel model of tele-assessment that has the potential for increasing access to services when barriers to in-person assessment exist. By quickly converting diagnostic evaluations to a telehealth platform, clinicians were able to continue to meet families' needs for timely access to ASD assessment and intervention services while considering family preference, clinician safety, and a societal obligation to social distance. The providers who participated in the piloting of this model shared qualitative feedback on the benefits and barriers to telemedicine evaluations, which has informed adjustments to our tele-assessment model over time. This paper also provides preliminary data on the use of the TELE-ASD-PEDS as a tool for telemedicine-based 
ASD evaluation in the home setting. This rapid response to the COVID-19 pandemic provides initial support and guidance for a re-conceptualization of ASD assessment, utilizing a novel tele-assessment tool that is both flexible and family-centered, and undoubtedly needed even beyond the pandemic.

Providers reported several perceived benefits to teleassessment. Beyond the opportunity to continue clinical services while following social distance mandates, providers noted that this model reduced family travel burden and increased access for families in rural or geographically isolated areas. Providers felt comfortable coaching parents through administration of the TELE-ASD-PEDS and providing diagnostic feedback to families via telemedicine. Providers also reported that it was beneficial to observe children and families in their home environments, interacting with familiar people and materials. Past research conducted in a laboratory setting has suggested that caregivers report tele-assessment to be comfortable and convenient, and that caregivers enjoy taking a more active role in the evaluation process (Corona et al. 2020b). Though caregiver feedback was not available for all families represented in the present study, evaluation of family satisfaction with clinical teleassessment is ongoing and will be an essential question for future research.

In addition to benefits of tele-assessment, providers identified several key barriers, with all providers endorsing technology-related challenges in their tele-assessment visits. This is a finding consistent with prior research on telehealthdelivered services (Juárez et al. 2018). Families were unfamiliar with the required technology (software requirements, apps) and often had difficulty navigating the EMR system to begin the visit or access their evaluation reports. Others had unstable wireless connections resulting in spotty audio and video. Finally, many caregivers had a difficult time filming their child in a way that allowed the remote provider to observe key behaviors (e.g., coordinated eye contact). It is possible that these technology-related challenges, when they did occur, had an impact on caregiver comfort with the evaluation process and confidence in the findings. In addition, distractions in the home environment sometimes impacted a providers' ability to gain a clear picture of the child's functioning or successfully communicate with caregivers and coach them through the assessment process.

These challenges prompted our clinical team to regularly revisit and adapt our internal processes for preparing families for appointments and communicating with families during and following tele-assessment. In response to the challenges described above, providers and staff within our medical center setting adjusted scheduling and communication procedures to prepare families for tele-assessment appointments. With regards to scheduling, a script was created for schedulers to use when contacting families. This script provided details about the tele-assessment visit, including technology requirements, caregiver involvement, and recommendations for optimal set-up (limiting distractions in the environment, suggested materials). All families had the option to decline tele-assessment and remain on the waitlist for in-person testing.

Due to the unique considerations presented by direct-tohome, caregiver-mediated telehealth assessment, providers found it valuable to have an open discussion at the outset about each person's role in the assessment process, possible outcomes, and limits to confidentiality. In an attempt to further mitigate technology-related barriers, some providers found it helpful to also use this conversation to provide a detailed overview of activities and examples of child behaviors we would be observing (e.g., eye contact, gestures, and facial expressions). Additionally, providers took this time to experiment with different camera placements at the beginning of the visit as opposed to adjusting after the evaluation was underway. Providers also increased their unstructured observations by asking caregivers to position their cameras on their children while answering interview questions.

With families for whom diagnostic uncertainty remained following tele-assessment, either due to technology problems or child-related factors, and for those families who explicitly requested further assessment, it was important to establish and explain a clear a mechanism through which families would be seen for additional in-person testing. Regardless of diagnosis issued, providers shared impressions with the family during the tele-assessment visit and offered information regarding services and resources that addressed child-specific concerns. Understanding the importance of balancing ease of access with HIPAA compliance, providers found it helpful to offer caregivers a choice between receiving documentation and resources via the EMR or through a secure email platform. Hard copies of reports were mailed if requested by families.

The transition to a tele-assessment model has also allowed for initial evaluation of the TELE-ASD-PEDS for clinical use. Though the TELE-ASD-PEDS is the focus of ongoing research in controlled settings (clinicaltrials.gov, NCT03847337), the present data represents the first use of this tool within families' homes. Within this sample, scores on the TELE-ASD-PEDS differed significantly in relation to providers' diagnostic impressions. As information gained from the TELE-ASD-PEDS was used to help form diagnostic impressions, it is not surprising that the TELE-ASDPEDS scores were highest for the group of children that received an ASD diagnosis (ASD Diagnosed), followed by children for whom providers suspected ASD but deferred a diagnosis pending further, in-person testing. Further, scores on the TELE-ASD-PEDS emerged as a significant predictor of diagnostic certainty. Providers were most confident in their impressions when issuing an ASD diagnosis. This 
provides preliminary support for use of this tool to confirm the presence of ASD via tele-assessment when there is identified risk. However, providers were less confident in their clinical impressions when there was diagnostic ambiguity and when ASD was ruled out, suggesting that further work is necessary to establish the utility of this tool to both identify ASD in children with more complex profiles, and to confidently rule out ASD.

\section{Limitations and Future Directions}

The present study provides initial information regarding the utility and provider acceptability of telemedicine-based ASD assessment. Though feasibility and acceptability are important first steps, rigorous empirical evaluation on the accuracy and effectiveness of these innovative methods is critical. Prior work has demonstrated that many young children with ASD can be accurately identified using clinic-based teleassessment procedures (Juárez et al. 2018), but it is unclear how diagnostic accuracy varies within direct-to-home care. With regards to the TELE-ASD-PEDS, there is currently a large study underway examining the psychometric properties of the tool relative to traditional, gold-standard evaluations (1R21MH118539-01), and initial data support the diagnostic accuracy of the TELE-ASD-PEDS when implemented in a laboratory setting (Corona et al. 2020b). However, we have not yet validated the TELE-ASD-PEDS when administered in home settings, introducing the risk that we are missing children who should have received a diagnosis, or providing an inaccurate diagnosis.

The current sample is weighted toward children who received an ASD diagnosis, due to our clinic-referred population of children already flagged as at-risk for ASD. There is a critical need for research evaluating the accuracy, validity, and functionality of a home-based TELE-ASD-PEDS when used with a larger, more heterogeneous sample of children. In particular, it will be important to understand for whom the TELE-ASD-PEDS - and tele-assessment more generally - are most effective. In the present study, clinicians reported highest level of diagnostic certainty for children with clear symptoms of ASD. More research is needed to determine the value of telemedicine evaluations for children with more complex profiles, including comorbid medical or developmental diagnoses.

Though direct-to-home telehealth services offer the potential for increased access to ASD-related assessment and services, not all families are comfortable with or appropriate for tele-assessment. Unfortunately, under-resourced families who are already disproportionately impacted by barriers related to geography, travel, and the time required for in-person evaluations may also lack access to the technology necessary for telemedicine appointments, such as stable internet connections and devices with audio-visual capabilities. In future work, it will be important to better understand the characteristics of families who either decline or are not eligible for these services to understand both barriers and facilitators to participation in tele-assessment. In addition, though preliminary research is in progress to assess caregiver satisfaction with teleassessment, future research will need to address caregiver perceptions of tele-assessment more systematically. Important questions remain regarding family satisfaction with the tele-assessment process and diagnostic outcomes, as well as the longitudinal impact of telemedicine evaluations on caregiver self-efficacy and child service eligibility. It is also unknown the extent to which complications caused by COVID-19 impacted families' decision to pursue (or refuse) tele-assessment.

Finally, the present study includes data only from providers employed at the site at which the TELE-ASD-PEDS was developed. Many of the providers were involved in the tool's development, and the majority have used the tool in research prior to changes resulting from the pandemic. Future work will assess provider perceptions and comfort at outside institutions and practices.

In conclusion, the unique set of circumstances created by the COVID-19 pandemic has allowed healthcare and behavioral health fields to trial telehealth approaches to patient care on a larger scale than ever before. The present study describes a model of telemedicine-based assessment for young children with concerns for ASD and demonstrates the potential utility of this approach for identifying children with clear ASD symptoms. The need for novel approaches to ASD assessment, particularly for under-resourced and geographically isolated families, existed before the pandemic and will likely continue after it. The lessons learned from adapting clinical services to reach families during this global crisis may well inform approaches to increasing service access for the future.

Author Contributions LW wrote the manuscript with support from LC, $\mathrm{AW}$, and $\mathrm{ZW}, \mathrm{LC}$ performed the statistical analyses. $\mathrm{KM}$ and $\mathrm{AB}$ created and maintained thedatabase for patient and provider data. LW, LC, $\mathrm{NB}, \mathrm{SF}, \mathrm{JH}, \mathrm{AN}, \mathrm{CS}$, and ZW contributed data from telehealth evaluations and provided theirperceptions of the tele-assessment model.

Funding The study was supported by funding from NIH/NIMH (R21MH118539), the Eunice Kennedy Shriver National Institute of Child Health and Human Development (U54 HD08321), and the Vanderbilt Institute for Clinical and Translational Research. The Vanderbilt Institute for Clinical and Translational Research (VICTR) is funded by the National Center for Advancing Translational Sciences (NCATS) Clinical Translational Science Award (CTSA) Program, Award Number 5UL1TR002243-03.

\section{Compliance with Ethical Standards}

Conflict of Interest Liliana Wagner, Laura Corona, Amy Weitlauf, Jeffrey Hine, Amy Nicholson, Caitlin Stone, and Zachary Warren are all 
co-authors of the TELE-ASD-PEDS. They do not receive compensation for the use of this instrument.

Ethics Approval All procedures performed in this study were in accordance with the ethical standards of the institutional research committee and with the 1964 Helsinki Declaration and its later amendments or comparable ethical standards. Analysis of existing clinical data was approved by the Institutional Review Board at Vanderbilt University Medical Center.

\section{References}

Adiani, D., Schmidt, M., Wade, J., Swanson, A. R., Weitlauf, A., Warren, Z., \& Sarkar, N. (2019). Usability enhancement and functional extension of a digital tool for rapid assessment of risk for autism spectrum disorders in toddlers based on pilot test and interview data. In International Conference on HumanComputer Interaction (pp. 13-22). Springer, Cham. https://doi. org/10.1007/978-3-030-23563-5_2

Antezana, L., Scarpa, A., Valdespino, A., Albright, J., \& Richey, J. A. (2017). Rural trends in diagnosis and services for autism spectrum disorder. Frontiers in Psychology, 8, 590. https://doi.org/10.3389/ fpsyg.2017.00590

Bishop-Fitzpatrick, L., \& Kind, A. J. (2017). A scoping review of health disparities in autism spectrum disorder. Journal of Autism and Developmental Disorders, 47(11), 3380-3391. https://doi. org/10.1007/s10803-017-3251-9

Clinicaltrials.gov, U.S. National Library of Medicine. (2019). Can novel telemedicine tools reduce disparities related to early identification of autism. Identifier NCT03847337. Retrieved February 1, 2020 from: https://clinicaltrials.gov/ct2/show/NCT03847337

Corona, L., Hine, J., Nicholson, A., Stone, C., Swanson, A., Wade, J., et al. (2020). TELE-ASD-PEDS: a Telemedicine-based ASD Evaluation Tool for Toddlers and Young Children. Vanderbilt University Medical Center. https://vkc.vumc.org/vkc/triad/ tele-asd-peds

Corona, L. L., Weitlauf, A. S., Hine, J., Berman, A., Miceli, A., Nicholson, A., et al. (2020). Parent perceptions of caregiver-mediated telemedicine tools for assessing autism risk in toddlers. Journal of Autism and Developmental Disorders. https://doi.org/10.1007/ s10803-020-04554-9

Corsello, C. M., Akshoomoff, N., \& Stahmer, A. C. (2013). Diagnosis of autism spectrum disorders in 2-year-olds: a study of community practice. Journal of Child Psychology and Psychiatry, 54(2), 178-185. https://doi.org/10.1111/j.1469-7610.2012.02607.x

Delacre, M., Leys, C., Mora, Y. L., \& Lakens, D. (2019). Taking parametric assumptions seriously: arguments for the use of Welch's F-test instead of the classical F-test in one-way ANOVA. International Review of Social Psychology. https://doi.org/10.5334/ irsp. 198

Durkin, M. S., Maenner, M. J., Meaney, F. J., Levy, S. E., DiGuiseppi, C., Nicholas, J. S., et al. (2010). Socioeconomic inequality in the prevalence of autism spectrum disorder: evidence from a US cross-sectional study. PLoS One, 5(7), e11551. https://doi. org/10.1371/journal.pone.0011551

Hyman, S. L., \& Johnson, J. K. (2012). Autism and pediatric practice: toward a medical home. Journal of Autism and Developmental Disorders, 42(6), 1156-1164. https://doi.org/10.1007/s 1080 3-012-1474-3

Joint Task Force for the Development of Telepsychology Guidelines for Psychologists. (2013). Guidelines for the practice of telepsychology. American Psychologist, 68(9), 791-800. https:// doi.org/10.1037/a0035001

Juárez, A. P., Weitlauf, A. S., Nicholson, A., Pasternak, A., Broderick, N., Hine, J., et al. (2018). Early identification of ASD through telemedicine: potential value for underserved populations. Journal of Autism and Developmental Disorders, 48(8), 2601-2610. https ://doi.org/10.1007/s10803-018-3524-y

Kanne, S. M., \& Bishop, S. L. (2020). Editorial perspective: the autism waitlist crisis and remembering what families need. Journal of Child Psychology and Psychiatry. https://doi.org/10.1111/ jcpp. 13254

Khowaja, M. K., Hazzard, A. P., \& Robins, D. L. (2015). Sociodemographic barriers to early detection of autism: screening and evaluation using the M-CHAT, M-CHAT-R, and follow-up. Journal of Autism and Developmental Disorders, 45(6), 1797-1808. https:// doi.org/10.1007/s10803-014-2339-8

Lord, C., Rutter, M., DiLavore, P. C., Risi, S., Gotham, K., \& Bishop, S. (2012). Autism diagnostic observation schedule, second edition (ADOS-2) manual (part 1): modules 1-4. Torrance, CA: Western Psychological Services.

Maenner, M. J., Shaw, K. A., Baio, J., Washington, A., Patrick, M., DiRienzo, M., et al. (2020). Prevalence of autism spectrum disorder among children aged 8 Years-autism and developmental disabilities monitoring network, 11 Sites, United States, 2016. Morbidity and Mortality Weekly Report: Surveillance Summaries, 69(4), 1-12. https://doi.org/10.15585/mmwr.ss6904a1

Mazurek, M. O., Handen, B. L., Wodka, E. L., Nowinski, L., Butter, E., \& Engelhardt, C. R. (2014). Age at first autism spectrum disorder diagnosis: the role of birth cohort, demographic factors, and clinical features. Journal of Developmental \& Behavioral Pediatrics, 35(9), 561-569. https://doi.org/10.1097/DBP.0000000000000097

Narzisi, A. (2020). Phase 2 and later of COVID-19 lockdown: is it possible to perform remote diagnosis and intervention for autism spectrum disorder? An online-mediated approach. Journal of Clinical Medicine, 9(6), 1850. https://doi.org/10.3390/jcm90 61850

Pierce, K., Gazestani, V. H., Bacon, E., Barnes, C. C., Cha, D., Nalabolu, S., et al. (2019). Evaluation of the diagnostic stability of the early autism spectrum disorder phenotype in the general population starting at 12 months. JAMA Pediatrics, 173(6), 578-587.

Stainbrook, J. A., Weitlauf, A. S., Juárez, A. P., Taylor, J. L., Hine, J., Broderick, N., et al. (2019). Measuring the service system impact of a novel telediagnostic service program for young children with autism spectrum disorder. Autism, 23(4), 1051-1056. https://doi. org/10.1177/1362361318787797

Wong, C. A., Ming, D., Maslow, G., \& Gifford, E. J. (2020). Mitigating the impacts of the COVID-19 pandemic response on at-risk children. Pediatrics, 145(4), e20200973. https://doi.org/10.1542/ peds.2020-0973

Wright, A. J., Mihura, J. L., Pade, H., \& McCord, D. M. (2020). Guidance on psychological tele-assessment during the COVID-19 crisis. Retrieved from https://www.apaservices.org/practice/reimb ursement/health-codes/testing/tele-assessment-covid-19

Zwaigenbaum, L., \& Warren, Z. (2020). Commentary: embracing innovation is necessary to improve assessment and care for individuals with ASD: a reflection on Kanne and Bishop (2020). Journal of Child Psychology and Psychiatry. https://doi.org/10.1111/ jcpp.13271

Publisher's Note Springer Nature remains neutral with regard to jurisdictional claims in published maps and institutional affiliations. 